ARTIGO

Recebido em: 12/01/2018

Aceito em: 08/05/2018

\title{
As variações terminológicas no contexto dos arquivos pessoais: uma análise das denominações utilizadas por instituições públicas brasileiras
}

The terminological variations in the context of personal papers: an analysis of the denominations used by brazilian public institutions

\author{
Bruna Pimentel LOPES (bpimentelopes@gmail.com)* \\ Georgete Medleg RODRIGUES (medleg.georgete@gmail.com) ** \\ * Doutoranda do Programa de Pós-Graduação em Ciência da Informação da Universidade de Brasília - \\ PPGCINF/UnB. \\ ** Doutora em História pela Université de Paris (Paris IV - Sorbonne). Professora do Curso de \\ Graduação em Arquivologia e do Programa de Pós-Graduação em Ciência da Informação da \\ Universidade de Brasília - UnB.
}

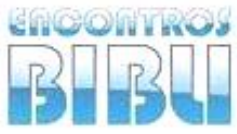

v. 23, n. $53,2018$. p. 87-95

ISSN $1518-2924$

\section{Resumo}

Considerando-se a frequência do uso de termos como "coleções", "papéis pessoais" e "manuscritos" para representar os arquivos pessoais, este artigo tem como objetivo identificar quais são os termos utilizados por entidades públicas brasileiras, mantenedoras de arquivos pessoais de escritores, para se referir a esse tipo de acervo. Para tanto, utiliza-se o método de pesquisa documental e a análise dos dados mediante estratégia comparativa de vinte instituições públicas. Verificou-se a existência de onze denominações distintas utilizadas pelas entidades analisadas para designar os arquivos pessoais de escritores, o que permite concluir a existência não só de diversas práticas para o tratamento desses acervos, mas uma dificuldade em percebê-los como conjuntos documentais arquivísticos.

Palavras-chave: Arquivos pessoais. Arquivos pessoais de escritores. Entidades custodiadoras de acervos arquivísticos. Terminologia arquivística.

\begin{abstract}
Considering the frequency of using terms such as "collections", "personal papers" and "manuscripts" to represent personal archives, this article aims to identify the terms used by brazilian public entities, maintainers of personal archives of writers, to refer to this type of collection. The method of documentary research and the analysis of data using a comparative strategy of twenty public institutions are used. It was verified the existence of eleven different denominations used by the entities analyzed to designate the personal archives of writers, which allows to conclude the existence not only of several practices for the treatment of these collections, but a difficulty in perceiving them as archival documentary sets.
\end{abstract}

Keywords: Personal archives. Personal archives of writers. Entities custodian of archival collections. Archival terminology.

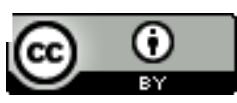

sta obra está licenciada sob uma Licença Creative Commons 


\section{INTRODUÇÃO}

Cox (2017), no livro intitulado "Arquivos pessoais: um novo campo profissional: leituras, reflexões e reconsiderações", afirma que o interesse em manter e preservar arquivos pessoais refere-se a questões que perpassam a memória coletiva, a missão dos profissionais que lidam com esse tipo de acervo, o papel dos arquivos institucionais e a função do indivíduo como seu próprio arquivista.

Dentro da perspectiva brasileira, a relevância desses acervos, tendo em vista seu caráter informacional, foi percebida recentemente, na década de 1970. De acordo com Costa (2006), tal fato decorre, principalmente, da busca de pesquisadores por fontes de informação de caráter privado. Segundo a autora, dentre os centros de documentação que surgiram no Brasil nesse contexto, destacam-se o Centro de Pesquisa e Documentação de História Contemporânea do Brasil (CPDOC), a Casa de Oswaldo Cruz, o Instituto de Estudos Brasileiros (IEB) e a Fundação Casa de Rui Barbosa (FCRB), entidades especializadas na custódia de fundos arquivísticos pessoais e que preencheram, ainda, uma lacuna decorrente da falta de uma política nacional de arquivos, formalizada posteriormente na década de 1990.

Para Heymann (2012), apesar do reconhecimento da importância de arquivos pessoais, há uma carência de estudos relacionados à temática no contexto da disciplina Arquivística que, muitas vezes, restringiu as reflexões teóricas do campo às práticas desenvolvidas no âmbito dos arquivos públicos. Conforme a autora, esta problemática pode ser percebida nas definições - muitas vezes restritivas - desses arquivos, como "o que não é público, o que não é institucional" (HEYMANN, 2012, p. 39).

Camargo (2009), por sua vez, chama-nos a atenção para o uso da expressão "arquivos pessoais" pela comunidade arquivística brasileira. Para a pesquisadora, o mais correto seria a utilização do termo "arquivos de pessoas", por ater-se a um determinado sujeito, de forma individual, ou de categorias ocupacionais, ou seja, arquivos de literatos, estadistas e cientistas.

Cabe ressaltar, inclusive, as diversas formas utilizadas para expressar "o conjunto de documentos produzidos e acumulados por uma pessoa ou família no desempenho de suas atividades" (OLIVEIRA, 2012, p. 31). Por terem sido tratados durante muito tempo a partir das técnicas biblioteconômicas, os arquivos pessoais são constantemente denominados como "coleções", "manuscritos" e "papéis pessoais". De acordo com Oliveira (2012) cada uma dessas categorias representa, de alguma forma, a incompreensão de que esses conjuntos documentais são arquivos e que, na maioria das vezes, são compostos por documentos em diferentes tipos de suporte.

Tal variedade terminológica - especialmente, a diversidade de termos utilizados para representar os arquivos pessoais - compreende a realidade não apenas internacional, mas também brasileira, e foi constatada quando do levantamento de dados de uma pesquisa de mestrado em Ciência da Informação que investigava se as diferentes formas de aquisição de arquivos pessoais de escritores custodiados por entidades brasileiras influenciariam as regras de acesso e uso desses acervos.

0 corpus do presente estudo compreende, portanto, vinte entidades públicas brasileiras (sete estados e o Distrito Federal) mantenedoras de arquivos pessoais de escritores para identificar quais são os termos que elas utilizam para representar esse tipo de acervo. 0 artigo está estruturado nas seguintes seções: a primeira contextualiza o problema a ser explorado no decorrer da pesquisa; a segunda aborda os procedimentos metodológicos utilizados; a terceira expõe as concepções em torno dos arquivos pessoais; a quarta apresenta os dados coletados durante a investigação e a sua análise; por fim, nas considerações finais, são relacionadas as contribuições trazidas pela pesquisa.

\section{PROCEDIMENTOS METODOLÓGICOS}

A análise da temática proposta foi feita a partir de uma abordagem qualitativa, uma vez que conforme MacDonald \& Headlam (2010) esta estratégia atém-se à compreensão das causas que motivam a ocorrência de determinado fenômeno. Nesse caso, pretendeu-se realizar um levantamento sobre a terminologia adotada pelas instituições públicas detentoras de arquivos pessoais de escritores para designar esse tipo de conjunto documental. 
A fim de alcançar este objetivo, foram levantados, inicialmente, órgãos públicos de interesse para a pesquisa de acordo com as informações obtidas por meio do Cadastro Nacional de Entidades Custodiadoras de Acervos Arquivísticos, ferramenta instituída por resolução do Conselho Nacional de Arquivos (CONARQ) ${ }^{1}$, que visa permitir o acesso às informações sobre a missão e o acervo, bem como os contatos dessas entidades.

Por meio de consultas na plataforma do CONARQ fez-se a seleção de entidades públicas localizadas nas capitais do Brasil que detêm arquivos pessoais de escritores. Além disso, tendo em vista o crescimento desse tipo de acervo em universidades, tal como é observado por Marques (2015), identificou-se instituições de ensino superior públicas que também mantêm esse tipo de arquivo. Desse modo, analisou-se a terminologia utilizada por vinte entidades de sete estados e do Distrito Federal. As informações foram coletadas dos sites dessas instituições e nas demais fontes documentais disponíveis online a fim de dar maiores subsídios para o estudo, aplicando, para tanto, o método de pesquisa documental 2 .

Para a fase de coleta de dados foi necessário, ainda, o uso de alguns instrumentos. No que diz respeito à pesquisa documental, o fichamento é uma ferramenta importante para a organização dos dados encontrados. 0 emprego deste tipo de recurso pressupõe, por exemplo, programas de bancos de dados localizados em computadores com o propósito de que as informações sejam armazenadas em um local apropriado (KAUARK, 2010). Assim, produziu-se uma planilha eletrônica com os dados referentes ao estado da federação em que as instituições estão localizadas, o nome do órgão e a terminologia utilizada para definir os arquivos pessoais.

Tendo em vista o problema de pesquisa, a análise das informações coletadas adotou um parâmetro comparativo entre as entidades para que fosse possível verificar a variedade de denominações utilizadas para representar esse tipo de acervo em âmbito nacional.

\section{OS ARQUIVOS PESSOAIS: uma revisão conceitual e terminológica}

Conforme definição apresentada no Dicionário de Terminologia Arquivística, publicado pelo Arquivo Nacional em 2005, os arquivos são um "conjunto de documentos produzidos e acumulados por entidade coletiva, pública ou privada, pessoa ou família, no desempenho de suas atividades" e que independem do tipo de suporte. De acordo com Lodolini (1993), para que sejam considerados documentos de arquivo, é necessário que esses registros tenham sido gerados no decurso de alguma atividade administrativa, empresarial, jurídica, pessoal e familiar.

Os acervos $^{3}$ arquivísticos representam, portanto, a organização sistemática de documentos originários de entidades produtoras de documentos, de acordo com um processo natural e com a sua ordem primária, a fim de que possam ser utilizados posteriormente como testemunho ou fonte de informação (HERRRERA, 1991). Assim, os arquivos distinguem-se das coleções, pois não são resultado de um ato voluntário e não reúnem documentos de maneira artificial, nem intencional, mas, primordialmente, de forma orgânica, conforme sua origem e finalidade (BELLOTTO, 2006; HERRERA, 1991).

Além disso, os arquivos diferenciam-se dos acervos bibliográficos e museológicos pois são produzidos exclusivamente por uma repartição, firma, pessoa ou instituição no decurso de suas atividades. Desse modo, uma das características essenciais dos conjuntos documentais arquivísticos consiste no elo que permite a ligação e a relação entre os registros que os compõem (PAES, 2004). Tal concepção refere-se à ideia de fundo, atribuída ao "conjunto de documentos de uma mesma proveniência", ou ainda, ao princípio de respeito aos fundos, segundo o qual "o arquivo produzido por uma entidade coletiva, pessoa ou família não deve ser misturado aos arquivos de outras entidades produtoras" (ARQUIVO NACIONAL, 2005, p. 97; 136).

\footnotetext{
1 Diz respeito à Resolução no 28, de 17 de fevereiro de 2009, disponível em: <http://conarq.gov.br/index.php/resolucoes-do-conarq/270-resolucao-n-28,-de-17-de-fevereiro-de-2009>. Acesso em: 07 jan. 2018.

2 De acordo com Payne \& Payne (2004) este método refere-se às técnicas utilizadas para categorizar, investigar, interpretar e identificar fontes físicas de informação, ou seja, avaliar documentos cujo conteúdo seja de interesse para o pesquisador

${ }^{3}$ Os acervos referem-se aos "documentos de uma entidade produtora ou de uma entidade custodiadora" (ARQUIVO NACIONAL, 2005, p. 19).
} 
No que se refere aos arquivos privados, esses acervos concernem aos registros de "uma entidade coletiva de direito privado, família ou pessoa" e podem ser denominados, inclusive, de arquivo particular (ARQUIVO NACIONAL, 2005, p. 35), ou seja, referem-se aos documentos privados ${ }^{4}$ produzidos por instituições não governamentais ou indivíduos, o que implica "considerar os arquivos econômicos ${ }^{5}$, os arquivos sociais ${ }^{6}$ e os arquivos pessoais" (BELLOTTO, 2006, p. 254).

Fraiz (1998) afirma que "foi somente a partir da segunda metade deste século ${ }^{7}$ que $o$ arranjo de papéis privados deixou de se basear em práticas e métodos biblioteconômicos" (FRAIZ, 1998, p. 62). Nos Estados Unidos, por exemplo, esses documentos eram tratados pelos bibliotecários por constituírem-se em peças avulsas e desorganizadas, o que os aproximava tecnicamente das coleções (COSTA, 2006).

Cook (1998), por sua vez, considera, inclusive, que nos Estados Unidos e na Austrália há a tradição dos manuscritos históricos versus a tradição dos arquivos públicos, segundo o qual os arquivistas de instituições públicos "ignoram" os colegas que tratam manuscritos, não os considerando como profissionais do ramo arquivístico, e sim, mais próximos das atividades biblioteconômicas e museológicas. Para o autor, em boa parte da Europa, os arquivos nacionais não reconhecem documentos pessoais de indivíduos particulares, padrão que se repete em arquivos estaduais, regionais e municipais. Tal prática evidencia que os arquivos pessoais, muitas vezes, são adquiridos por bibliotecas nacionais, regionais, museus ou universidades. Essa variedade de domicílios institucionais para conjuntos documentais produzidos no âmbito de arquivos públicos ou em ambientes domésticos - no caso dos arquivos pessoais - reafirmam suas diferenças.

Apesar disso, segundo Costa (2006) com o crescimento desses conjuntos documentais e a comprovação de que possuíam relação orgânica, atualmente os profissionais que lidam com arquivos pessoais têm recorrido aos métodos arquivísticos para o seu tratamento e organização.

Dentro desta perspectiva, esses acervos podem ser entendidos como um conjunto de documentos produzidos e mantidos por um indivíduo ao longo de sua vida, ou seja, tratamse de registros que representam suas atividades profissionais e pessoais, e concernem, em geral, aos "arquivos de homens e mulheres que se destacaram ao longo da história de uma sociedade" (OLIVEIRA, 2012, p. 39) ${ }^{8}$. Bellotto (2006), afirma que os arquivos pessoais trazem em sua definição a própria ideia de arquivos privados, uma vez que se constituem de registros produzidos e/ou recebidos por pessoas físicas - na maioria das vezes artistas, políticos, literatos, cientistas, etc. - relacionados à sua vida profissional e familiar e que são preservados, do ponto de vista institucional, considerando-se o interesse para a pesquisa histórica e o ineditismo das informações neles contidas. Segundo Heymann (2012), em geral, a disciplina arquivística caracteriza os titulares desses acervos como "estadistas", "políticos" "literatos" ou "cientistas", vinculando a identidade do produtor ao desempenho de uma determinada atividade profissional.

Conforme ressaltado por Silva (2017) a liberdade de acumulação é uma das principais características que constituem os arquivos pessoais, pois, neste caso, os indivíduos podem guardar documentos de acordo com os seus próprios desejos e critérios. Apesar disso, para Silva e Melo (2016) não faz sentido que os arquivos pessoais sejam compreendidos como peças individuais que não possuem relação entre si. Tampouco faz sentido tratar esses acervos como uma coleção, como se esses conjuntos documentais fossem resultados de processos artificiais.

Por outro lado, Oliveira (2012) observa quão diversas são as formas utilizadas para expressar esses conjuntos documentais, pois os arquivos pessoais foram tradicionalmente

\footnotetext{
4 "É o documento privado isolado, produzido ou recebido por entidade não-governamental ou pessoa física" (BELLOTTO, 2006, p. 250).

5 "Esta categoria compreende a documentação gerada por empresas comerciais, industriais, financeiras ou de serviços, de pequeno, médio ou grande portes" (BELLOTTO, 2006, p. 254).

6 "Abrangem um grande número de arquivos importantes, sobressaindo-se os religiosos, os notariais e os de movimentos e entidades políticas" (BELLOTTO, 2006, p. 254).

${ }^{7}$ Neste caso, refere-se ao século XX.

${ }^{8}$ No entanto, cabe ressaltar que, conforme Artières, "arquivar a própria vida não é privilégio de homens ilustres (de escritores ou de governantes). Todo indivíduo, em algum momento da sua existência, por uma razão qualquer, se entrega a esse exercício" (ARTIÈRES, 1998, p. 31). Trata-se, de acordo com o autor, de uma prática íntima que, muitas vezes, tem função pública.
} 
custodiados por bibliotecas e museus, sendo associados, portanto, às concepções de manuscritos, coleções ou papéis pessoais. Na literatura internacional - particularmente americana e canadense - "o termo manuscrito se refere aos papéis históricos ou literários dos arquivos pessoais ou de família, enquanto o termo arquivo (archives) diz respeito ao arquivo permanente de uma organização privada ou governamental" (OLIVEIRA, 2012, p. 31).

Nessa perspectiva a expressão "papéis pessoais" é utilizada somente quando diz respeito aos arquivos pessoais e familiares, o que representa não só a exclusão de diversos suportes da categoria, mas indica justamente a concepção errônea de que há uma falta de contexto e ligação entre esses documentos (OLIVEIRA, 2012). Por fim, ainda no caso dos Estados Unidos e do Canadá, "o termo coleção expressa o que se identifica conceitualmente como coleção, ou mesmo arquivo, no que se refere aos arquivos pessoais; não há distinção no uso dos termos" (OLIVEIRA, 2012, p. 32), pois, frequentemente, o conceito de arquivo simplesmente não é atribuído aos conjuntos documentais de pessoas. No caso do Reino Unido e da França são utilizadas as expressões personal archives ou archives personnelles, respectivamente (OLIVEIRA, 2012).

Tal variação terminológica indica, conforme Camargo e Goulart (2007) que os arquivos pessoais têm sido considerados como coleções de documentos ${ }^{9}$ e "abordados por meio de critérios originários das bibliotecas, coerentes com a tradição de ali se depositarem as obras e os demais papéis de escritores" (CAMARGO; GOULART, 2007, p. 37). Entretanto, as autoras afirmam que para analisar "arquivos pessoais como arquivos, é preciso vê-los, antes de tudo, como 'conjuntos solidários e orgânicos'” (CAMARG0; GOULART, 2007, p. 43).

Heymann (2012) atribui esta problemática ao lugar secundário que os arquivos pessoais ocupam nas discussões teóricas no âmbito da Arquivologia, afirmando, que:

\begin{abstract}
Muitas vezes tratados segundo princípios biblioteconômicos, integrados a seções de "manuscritos nas bibliotecas", os arquivos pessoais ocupam uma zona de fronteira, submetidos a abordagens metodológicas distintas, de acordo com as filiações institucionais e disciplinares dos responsáveis por sua custódia (HEYMANN, 2012, p. 53).
\end{abstract}

A inadequação terminológica também é reafirmada por Camargo (2009), que propõe o uso da terminologia arquivo de pessoas ou de categorias ocupacionais para designar os arquivos pessoais. Segundo a autora, o uso deste termo traz conflitos em três situações: a) por referir-se, ao mesmo tempo, aos documentos sobre pessoas que compõem os arquivos institucionais; b) como também aos registros acumulados por indivíduos; c) e às parcelas específicas dos arquivos, compostas por documentos identitários, tais como cédulas de identidade, títulos eleitorais e passaporte. A observação é válida, inclusive, para a ideia de que esses conjuntos documentais são constituídos apenas por uma das atividades desenvolvidas pelo titular e que são representadas por denominações como "arquivos científicos", "arquivos políticos", "arquivos religiosos", etc.

\title{
4 A DIVERSIDADE TERMINOLÓGICA PARA REPRESENTAR OS ARQUIVOS PESSOAIS
}

Tendo em vista o contexto brasileiro e as informações coletadas a partir da pesquisa documental nas vinte instituições públicas - localizadas em sete estados brasileiros e o Distrito Federal - custodiadoras de arquivos pessoais de escritores, observa-se no quadro a seguir os dados obtidos durante a investigação:

\footnotetext{
9 "Os autores clássicos da área arquivística dedicaram-se especialmente aos documentos acumulados por instituições, encarando os pessoais, quando muito, como complementares" (CAMARGO, GOULART, 2007, p. 37).
} 
Quadro 1 - Terminologia utilizada por instituições custodiadoras de acervos arquivísticos para representar arquivos pessoais de escritores

\begin{tabular}{|c|c|c|}
\hline ESTADO & INSTITUIÇÃO & TERMINOLOGIA \\
\hline \multirow{2}{*}{$\begin{array}{l}\text { DISTRITO } \\
\text { FEDERAL }\end{array}$} & Arquivo Público do DF & Fundo \\
\hline & Biblioteca Central da Universidade de Brasília & Fundo privado \\
\hline \multirow[b]{2}{*}{ BAHIA } & Centro de Memória da Bahia & Arquivo privado \\
\hline & $\begin{array}{l}\text { Núcleo de Arquivos Históricos Pessoais e } \\
\text { Institucionais da UFBA }\end{array}$ & Fundo pessoal \\
\hline \multirow{3}{*}{ MINAS GERAIS } & Arquivo Público da Cidade de Belo Horizonte & Acervo privado \\
\hline & Arquivo Público Mineiro & Fundo privado \\
\hline & Acervo de Escritores Mineiros - UFMG & Acervo \\
\hline PERNAMBUCO & Arquivo Público de Pernambuco & Arquivo particular \\
\hline \multirow{4}{*}{ SÃO PAULO } & Arquivo Público do Estado de São Paulo & Arquivo particular \\
\hline & Centro de Documentação e Memória da Unesp & Acervo textual privado \\
\hline & Centro de Documentação e Memória da Pinacoteca & Fundo \\
\hline & Instituto de Estudos Brasileiros & Fundo \\
\hline ESPÍRITO SANTO & Arquivo Público do Estado do Espírito Santo & $\begin{array}{l}\text { Fundo documental } \\
\text { privado }\end{array}$ \\
\hline \multirow{6}{*}{ RIO DE JANEIRO } & Arquivo Histórico do Museu da República & Coleção \\
\hline & $\begin{array}{c}\text { Arquivo-Museu de Literatura Brasileira - Fundação } \\
\text { Casa Rui Barbosa }\end{array}$ & Arquivo \\
\hline & Arquivo Nacional & Fundo \\
\hline & Biblioteca Nacional & Coleção \\
\hline & Casa de Oswaldo Cruz & Fundo \\
\hline & Centro de Cidadania Barbosa Lima Sobrinho & Acervo \\
\hline $\begin{array}{l}\text { RIO GRANDE DO } \\
\text { SUL }\end{array}$ & $\begin{array}{l}\text { Arquivo Histórico do } \\
\text { Rio Grande do Sul }\end{array}$ & Arquivo particular \\
\hline
\end{tabular}

Fonte: Elaboração própria com base nos dados obtidos na pesquisa.

As informações do Quadro 1 evidenciam que os arquivos pessoais têm sido objeto de investimento das mais diversas instituições. Destacam-se, assim, não só os arquivos, mas centros de documentação e bibliotecas vinculados às universidades. Conforme Santos (2012), a aquisição de arquivos pessoais por entidades variadas está atrelada ao reconhecimento da sua importância para fins históricos ou científicos. Desse modo, percebese que os arquivos públicos tradicionalmente têm adquirido esse tipo de acervo, "mas bibliotecas e museus, ligados ou não a [outras] instituições, como por exemplo, universidades, também possuam a guarda de importantes conjuntos documentais oriundos de pesquisadores e pessoas de relevância para a cultura nacional" (SANTOS, 2012, p. 90).

Cabe ressaltar, inclusive que, embora não sejam tratados, na maioria dos casos, por entidades arquivísticas, esses acervos são organizados a partir da perspectiva de fundo, ou seja, baseando-se na "teia de relações (originária, necessária e determinada) que os documentos de um mesmo grupo mantêm entre si" (CAMARGO; GOULART, 2007). Desse modo, os arquivos pessoais são muitas vezes caracterizados como "fundo privado", "fundo pessoal" e "fundo documental privado". Esta constatação pode ser mais bem visualizada no gráfico que se segue: 
Gráfico 1 - Variedade terminológica nas instituições pesquisadas

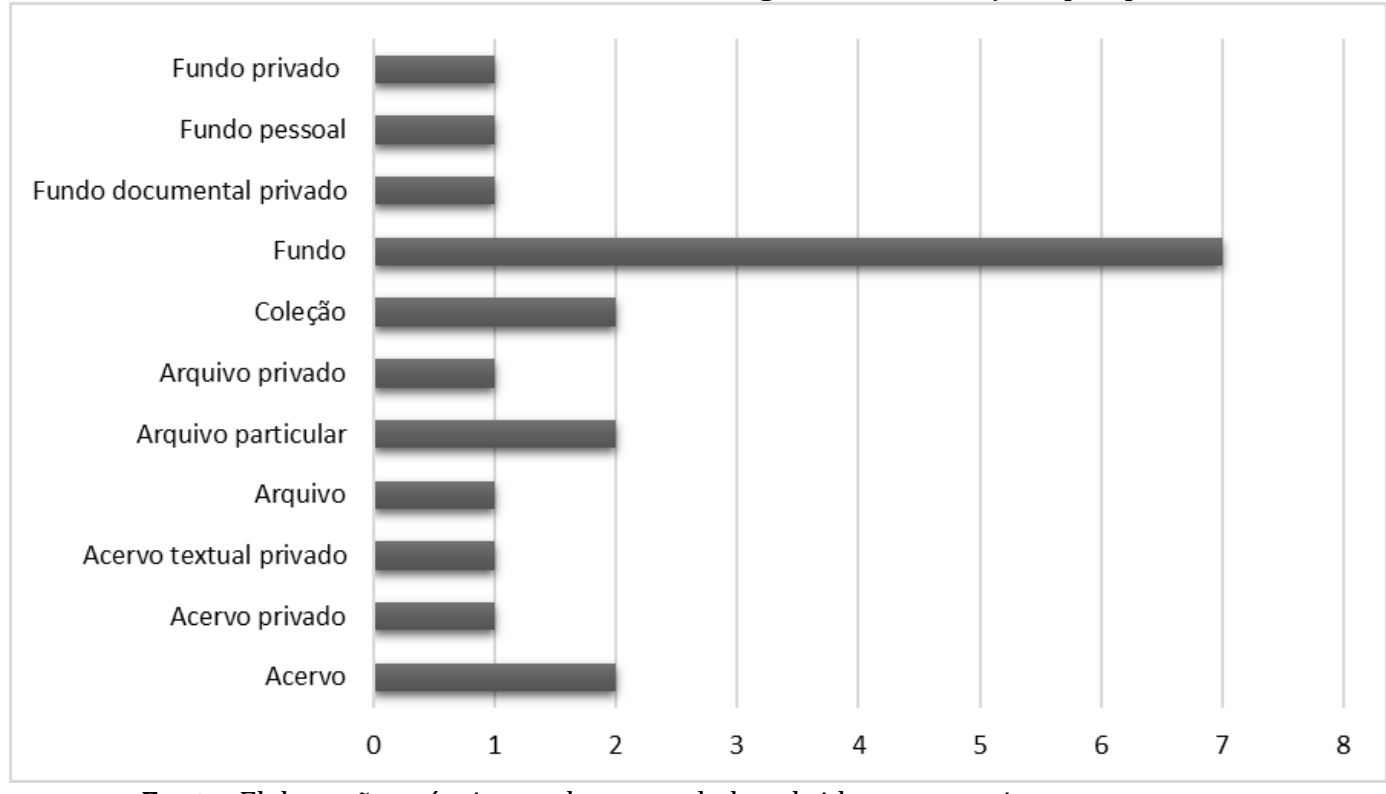

Fonte: Elaboração própria com base nos dados obtidos na pesquisa.

Nessa perspectiva, é importante destacar que, a partir da década de 1980, houve um impulso para a normalização da descrição arquivística em âmbito internacional. Alguns países como os Estados Unidos e a Inglaterra já haviam percebido tal necessidade, lançando, na década anterior, o formato MARC, específico para arquivos e manuscritos. Posteriormente, em 1983, Steven L. Hesen publicou o manual Archives, personal papers and manuscripts: a cataloguing manual for archival repositories, historical societies and manuscripts, conhecido pela sigla APPM, o que evidencia certa preocupação no tratamento de manuscritos pessoais localizados noutras instituições além dos arquivos (CONSELHO NACIONAL DE ARQUIVOS, 2006).

A Norma Brasileira de Descrição Arquivística (NOBRADE), por sua vez, publicada em 2000, que se baseia na Norma Geral Internacional de Descrição Arquivística (ISAD (g)), prevê a sua adoção para o tratamento de documentos pessoais custodiados por bibliotecas e museus, apresentando como exemplo a descrição de um fundo pessoal, o de José Feio, sob custódia do Museu Nacional.

Apesar disso, outro dado expressivo localizado durante a pesquisa demonstra que algumas entidades classificam esses acervos como coleções. Tal fato pode ser exemplificado pelo Arquivo Histórico do Museu da República ${ }^{10}$ e pela Biblioteca Nacional ${ }^{11}$, que detêm conjuntos documentais de escritores como Machado de Assis (1839-1908) e Euclides da Cunha (1866-1909), respectivamente, e consideram esses conjuntos documentais como coleção, independentemente da sua forma de produção e acumulação.

Nesse sentido, observa-se que muitas vezes os arquivos pessoais são submetidos a abordagens metodológicas distintas, apesar de a disciplina arquivística dispor de instrumentos para a padronização e unificação da terminologia da área. Ignorar a contribuição da Arquivologia nesse campo resulta, muitas vezes, em uma multiplicidade de tradições e práticas.

Tendo em vista que, de acordo com Bellotto (2006), esses conjuntos documentais correspondem a uma categoria dos arquivos privados, as informações coletadas evidenciam também que os profissionais das instituições que os custodiam designam tais acervos como "arquivo privado", "acervo privado", "arquivo particular" ou simplesmente "arquivo" ou "acervo". Conforme Heymann (2012), a aproximação entre os arquivos privados e pessoais baseia-se na natureza jurídica desses fundos, ou seja, no fato de serem produzidos por

${ }^{10}$ As informações referentes ao acervo deste Arquivo podem ser consultadas no Guia de Coleções, disponível em: <http://museudarepublica.museus.gov.br/guia-de-colecoes/\#indice19>. Acesso em: 09 jan. 2018.

11 Mais informações sobre as coleções mantidas pela Biblioteca Nacional podem ser consultadas no Catálogo de Manuscritos, disponível em: <http://acervo.bn.br/sophia_web/index.html>. Acesso em: 09 jan. 2018. 
entidades privadas e não pelo Estado. Entretanto, de acordo com a historiadora, “o pertencimento à categoria dos arquivos privados parece prevalecer em relação às especificidades da entidade produtora, sendo equiparados os processos de acumulação de documentos por entidades e indivíduos" (HEYMANN, 2012, p. 54).

Além disso, os resultados encontrados no presente estudo indicaram onze formas diferentes para representar os arquivos pessoais, o que vai ao encontro das afirmações de Oliveira (2012), segundo a qual esses acervos são categorizados de múltiplas formas pela comunidade arquivística. É possível afirmar, nesse caso, que tal variedade demonstra "a falta de investimentos específicos nos conjuntos de natureza pessoal por parte da comunidade arquivística, reflexo do lugar periférico que ocupam no universo dos arquivos" (HEYMANN, 2012, p. 52).

\section{CONSIDERAÇÕES FINAIS}

Ainda que o reconhecimento da importância das fontes arquivísticas de caráter privado - particularmente os registros pessoais - seja recente, os dados obtidos durante a pesquisa sinalizam que os arquivos pessoais têm sido adquiridos por instituições de natureza variada, tendo em vista seus aspectos informacionais.

Entretanto, a multiplicidade de profissionais responsáveis pelo tratamento desses acervos e, sobretudo, o longo período de resistência por parte dos arquivistas em relação ao tratamento desses conjuntos documentais, ocasionaram o surgimento de práticas distintas para a organização dos arquivos pessoais. Embora, atualmente, sejam desenvolvidas discussões para aprimorar o tratamento desses acervos, o reflexo desta constatação pode ser verificado nas diversas formas utilizadas para representá-los e categorizá-los.

Percebe-se que a Arquivologia tem buscado unificar e consolidar as suas práticas. Entretanto, no contexto dos arquivos pessoais, há uma carência de maiores investimentos. No caso da diversidade terminológica com a qual esses acervos são representados, é necessário que haja uma padronização mais eficaz a fim de proporcionar maior qualidade ao trabalho técnico e à recuperação e, consequentemente, ao acesso a essas informações. E, ainda, para que esses conjuntos documentais sejam, de fato, interpretados como arquivos.

Portanto, o panorama traçado pelo estudo, sinaliza a necessidade de novos debates em busca de uma padronização terminológica e, principalmente, um trabalho compartilhado, a ser realizado de maneira comum não apenas por arquivistas, mas por todos os profissionais do campo da informação - bibliotecários, museólogos - bem como os historiadores, que são, muitas vezes, responsáveis pelo tratamento, preservação e guarda dos arquivos pessoais.

Por fim, a pesquisa sugere que as próprias características intrínsecas aos arquivos pessoais - especialmente a liberdade de acumulação e seleção pelo produtor - ainda apresentam certas dificuldades para o tratamento documental. Fato que igualmente se reflete nas variações terminológicas utilizada para designá-los.

\section{REFERÊNCIAS}

ARQUIVO NACIONAL (BRASIL). Dicionário brasileiro de terminologia arquivística. Rio de Janeiro: Arquivo Nacional, 2005.

BELLOTTO, H. L. Arquivos permanentes: tratamento documental. 4. Ed. Rio de Janeiro: FGV, 2006.

CAMARGO, Ana Maria de Almeida. Arquivos pessoais são arquivos. Revista do Arquivo Público Mineiro, Belo Horizonte, v. 45, n. 2, p. 26-39, jul./dez. 2009.

CAMARGO, A. M. A.; GOULART, S. Tempo e circunstância: a abordagem contextual dos arquivos pessoais: procedimentos metodológicos adotados na organização dos documentos de Fernando Henrique Cardoso. São Paulo: Instituto Fernando Henrique Cardoso, 2007.

CONSELHO NACIONAL DE ARQUIVOS (BRASIL). NOBRADE: Norma Brasileira de Descrição Arquivística. Rio de Janeiro: Arquivo Nacional, 2006. 
COOK, T. Arquivos pessoais e arquivos institucionais: para um entendimento comum da formação da memória em um mundo pós-moderno. Estudos históricos, Rio de Janeiro, v. 11, n. 21, p. 129-150, 1998.

COSTA, C. M. L. A política nuclear nos arquivos pessoais. In: ABREU, A. A. A democratização no Brasil: atores e contextos. Rio de Janeiro: FGV, 2006.

COX, R. Arquivos pessoais: um novo campo profissional: leituras, reflexões e reconsiderações. Belo Horizonte: Editora UFMG, 2017.

FRAIZ, P. A. dimensão autobiográfica dos arquivos pessoais: o arquivo de Gustavo Capanema. Estudos Históricos, v. 11, n. 21, p. 59-88, 1998.

HERRERA, A. H. Archivistica general: teoria y pratica. Sevilla: Diputación Provincial de Sevilla, 1991.

HEYMANN, L. Q. 0 lugar do arquivo: a construção do legado de Darcy Ribeiro. Rio de Janeiro: Contracapa / FAPERJ, 2012.

KAUARK, F. Metodologia da pesquisa: guia prático. Itabuna: Via Litterarum, 2010.

LODOLINI, E. Archivística: princípios y problemas. Madrid: ANABAD, 1993.

PAES, M. L. Arquivo: teoria e prática. Rio de Janeiro: FGV, 2004.

MACDONALD, S.; HEADLAM, N. Research methods handbook: introductory guide to research methods for social research. United Kingdom: CLES, 2010.

MARQUES, R. Arquivos literários: teorias, histórias, desafios. Belo Horizonte: Editora UFMG, 2015.

OLIVEIRA, L. M. V. Descrição e pesquisa: reflexões em torno dos arquivos pessoais. Rio de Janeiro: Móbile, 2012.

PAYNE, G.; PAYNE, J. Key concepts in social research. London: SAGE Publications, 2004.

SILVA, E. P.; MELO, M. T. A dispersão de fundos de arquivos pessoais. Revista do Arquivo Geral da cidade do Rio De Janeiro, Rio de Janeiro, n.10, p.91-102, 2016.

SANTOS, P. R. E. dos. Arquivos de cientistas: gênese documental e procedimentos de organização. São Paulo: Associação de Arquivistas de São Paulo, 2012.

SILVA, M. C. S. M. E. Presentación: os arquivos pessoais e os desafios para os arquivistas. In: GARCIA, N.; SILVA, M. C. S. M.(Org.). Archivos personales: experiencias de organización y gestión. Córdoba: Editorial de la Red de Archiveros Graduados de Córdoba, 2017.

Editores do artigo: Enrique Muriel-Torrado, Edgar Bisset Alvarez, Camila Barros. 